\title{
Coopération politique et intégration régionale en Arctique : naissance, développement et critique d'une région
}

Political cooperation and regional integration in the Arctic: construction, development and challenging of a model

\section{Camille Escudé-Joffres}

\section{(2) OpenEdition}

Journals

\section{Édition électronique}

URL : http://journals.openedition.org/belgeo/43757

DOI : 10.4000/belgeo.43757

ISSN : 2294-9135

\section{Éditeur :}

National Committee of Geography of Belgium, Société Royale Belge de Géographie

\section{Référence électronique}

Camille Escudé-Joffres, « Coopération politique et intégration régionale en Arctique : naissance, développement et critique d'une région », Belgeo [En ligne], 4 | 2020, mis en ligne le 09 novembre 2020, consulté le 11 novembre 2020. URL : http://journals.openedition.org/belgeo/43757 ; DOI : https://doi.org/10.4000/belgeo.43757

Ce document a été généré automatiquement le 11 novembre 2020.

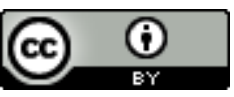

Belgeo est mis à disposition selon les termes de la licence Creative Commons Attribution 4.0 International. 


\title{
Coopération politique et intégration régionale en Arctique : naissance, développement et critique d'une région
}

\author{
Political cooperation and regional integration in the Arctic: construction, \\ development and challenging of a model
}

Camille Escudé-Joffres

\section{Introduction}

1 Jusqu'à la fin de la Guerre froide, l'Arctique était conçu dans la géopolitique mondiale comme un espace de potentiel affrontement entre deux blocs ennemis. Depuis, en l'espace de trente ans, une structure de gouvernance multilatérale a été construite $e x$ nihilo grâce à l'interaction de différents niveaux d'action politique correspondant à divers acteurs: États, société civile, organisations autochtones, gouvernements fédéraux et locaux, ONG. Au niveau régional, le Conseil de l'Arctique créé en 1996 est l'organisation prééminente de coopération politique. Auprès des huit États circumpolaires $^{1}$ de ce Conseil sont présents en 2020 six organisations autochtones et trente-neuf membres observateurs étatiques ou non-étatiques.

2 Au-delà des seules organisations régionales, l'Arctique comme espace est impliqué dans divers mécanismes politiques qui tendent vers une intégration, définie comme processus par lequel la régularité et l'intensité des interactions entre les sociétés s'accroissent. La manière dont la gouvernance à toutes les échelles a construit un réseau d'acteurs conduit à penser l'Arctique comme un système de gouvernance multiniveau. Nous sommes en effet invités ici à dépasser la conception westphalienne du territoire pour envisager une région en creux, comme niveau de médiation entre des hiérarchies traditionnellement constituées. Parce que ce sont des volontés politiques 
qui ont engagé le processus de gouvernance et ainsi permis l'intégration régionale, la région a des limites floues, mouvantes et contestées. Il s'agira de comprendre la région comme processus et non pas comme état, en faisant de l'espace arctique un enjeu de débat dans une perspective constructiviste.

\section{Cadre théorique : la région comme espace construit}

3 Entre les années 1980 et 1990, les théories du nouveau régionalisme ont renouvelé et enrichi les débats sur l'intégration régionale avec des travaux marqués par le constructivisme social. Pour les tenants du nouveau régionalisme, les régions sont le produit d'un processus de construction sociale et politique. Ce nouveau régionalisme naît dans le champ de la science politique. Mais comme l'écrit Yann Richard (2018, p. 184): «Les tenants du nouveau régionalisme croisent inévitablement la route des géographes puisque ces derniers ont montré depuis longtemps que l'espace géographique est une production sociale, tout en reconnaissant qu'il existe une interaction entre le social et le spatial: ce sont les sociétés qui produisent l'espace géographique (car elles se projettent de telle ou telle façon à la surface de la Terre); l'espace géographique n'est pas figé et est le résultat de la projection des sociétés humaines, elles-mêmes historiques; rétroactivement, l'agencement de l'espace géographique a une incidence sur les pratiques sociales ».

4 L'idée selon laquelle la région est une construction sociale, qui peut se faire et se défaire, est devenue peu à peu centrale dans de nombreux travaux géographiques, notamment à partir des années 1980 chez les tenants de la new regional geography adoptant une posture critique tels que John Agnew ou Anssi Paasi. Pour ces chercheurs, la région serait un projet intentionnel, un assemblage politique. Ainsi, dans leur ouvrage Rethiking the region (1998), John Allen, Doreen Massey et Allan Cochrane affirment ainsi que les régions ne sont pas de simples espaces bornés sur une carte. Elles ne possèdent pas non plus la même forme, la même géographie à travers le temps. Pour eux, les régions sont bien plutôt faites et refaites par des processus spatiaux qui les affectent de manière inégale. Alors que l'approche la plus répandue pour étudier la différentiation régionale est de cartographier l'absence et la présence de phénomènes dans l'espace, les auteurs affirment que l'espace social peut être aussi compris comme le produit de relations sociales: la création des régions est conditionnée par les politiques, les cultures, les économies, les gouvernances et les relations de pouvoir (Paasi, 2010).

5 À ce titre, l'existence des régions est précédée par celle de region-builders, des acteurs qui fabriquent les régions comme résultat d'un projet politique. Dans la lignée de ces travaux, nous allons nous attacher à démontrer comment le réseau de gouvernance né en Arctique aux lendemains de la Guerre froide participe à la création d'une région. $\mathrm{Si}$ la région est un outil politique, il s'agira de saisir les intentions des acteurs derrière la seule construction institutionnelle.

\section{Problématique}

6 La naissance et la définition des politiques du Conseil de l'Arctique sont étroitement liées à la protection de l'environnement et la promotion du développement durable, comme plus petit dénominateur commun pour rassembler les États circumpolaires à la 
fin de la Guerre froide. Dans un contexte de perception accrue des enjeux politiques et économiques dans les hautes latitudes depuis une vingtaine d'années, la forme actuelle de gouvernance semble mise à l'épreuve. De nouveaux acteurs qui font irruption dans la région critiquent le modèle actuel de gouvernance, réduit aux acteurs étatiques circumpolaires, alors que les enjeux arctiques se mondialisent du point de vue environnemental, économique et commercial. Dans ce double contexte d'ouverture internationale de la région arctique et de raidissement politique des États arctiques, les dynamiques centrifuges et centripètes questionnent la forme et les limites de cette région construite par un processus politique.

\section{Méthodologie}

7 Ce travail est fondé sur une étude à la fois théorique et empirique. D'une part, cet article s'appuie sur la littérature constructiviste sur la région et un travail d'analyse politique des structures de gouvernance arctique. D'autre part, les acquis originaux de cette publication sont issus de témoignages d'acteurs, interrogés directement ou fréquentés lors d'observations participantes. Notre étude empirique s'appuie ainsi sur quarante entretiens menés auprès de représentants des États arctiques, d'états extérieurs à la région mais souhaitant intervenir dans la gouvernance régionale, de représentants politiques autochtones ainsi que de membres d'ONG.

Plan

8 Nous émettons dans un premier temps l'hypothèse que c'est la coopération politique impulsée "par le haut» dans l'après-Guerre froide qui a conduit à l'intégration régionale institutionnelle visible en Arctique. Cette intégration institutionnelle est issue de la volonté étatique de protection de l'environnement comme prétexte à la coopération politique.

9 Il apparait alors que dans un contexte de politisation accrue de la région, la construction de la région arctique devient pour les États arctiques un moyen d'exclure certains acteurs : organisations autochtones et acteurs extérieurs sont progressivement poussés hors des instances décisionnelles. En retour, ces derniers élargissent les limites de la région à travers de nouvelles formes de gouvernance politique (forums et conférences).

10 Les acteurs politiques à différentes échelles convergent ainsi pour construire une gouvernance régionale qui n'est pas seulement multiniveau, mais encore enchevêtrée. Bien que cette intégration soit plus institutionnelle que fonctionnelle, les stratégies explicites ou implicites des gouvernances multiniveaux révèlent la formation d'une une région à la forme particulière.

\section{La région arctique, fruit d'une volonté politique impulsée « par le haut » dans l'après-Guerre froide}

À la fin de la Guerre froide, l'Arctique est devenu le théâtre d'une multitude de tentatives d'intégrations institutionnelles, dont la plus aboutie est le Conseil de l'Arctique. Ce dernier est issu de la volonté étatique de protection de l'environnement 
et de promotion du développement durable. Il apparaît cependant comme prétexte à la coopération politique dans une région fortement marquée par des tensions géopolitiques.

\section{Comment mesurer l'intégration régionale en Arctique ? L'aide des théories de la « régionalité »}

12 La question de la mesure de l'intégration régionale en Arctique est complexe. À partir de quel niveau d'interaction peut-on considérer qu'il y a une intégration et la construction d'un système régional? Comment peut-on mesurer ces interactions et avec quels indicateurs ? Cette difficulté se double du fait que, nulle part dans le monde, l'intégration régionale n'a atteint son terme théorique.

Le concept central chez Björn Hettne et Frederik Söderbaum, théoriciens de la région en tant qu'espace construit, est la regionness qu'on pourrait traduire en français par " régionalité ». Les deux auteurs définissent cinq niveaux de cette "régionalité " (Hettne, 1994; Hettne \& Söderbaum, 2000 ; Hettne, 2003) et mesurent l'intégration régionale à travers le passage d'un degré à l'autre. Les cinq niveaux sont les suivants : la région comme unité géographique et écologique ; la région comme système social plus ou moins conflictuel d'interactions culturelles politiques et économiques; la région comme mécanisme élémentaire de sécurité, coopération organisée en matière culturelle, économique, politique ou militaire institutionnalisée par des organisations régionales multilatérales; l'organisation régionale: la région comme société civile émergeant par la culture, la communication sociale et la convergence de valeurs; la société régionale produite par cette organisation: la région comme construction historique d'une entité distincte agissant comme acteur politique doté d'un certain niveau de légitimité.

Cette montée de degrés signifie que l'intégration est le processus par lequel une portion d'espace est peu à peu remplie par assez de «substance économique, institutionnelle, politique, culturelle et finalement identitaire pour devenir un système distinct des autres et être perçue comme tel " (Richard, 2018, 185). Au lieu de penser la région comme un simple état de fait, il est alors intéressant de penser le processus. Cela implique qu'une région peut être "plus ou moins" région et que ce niveau de « régionalité » peut évoluer dans le temps et connaître un processus réversible.

Selon cette typologie, la région arctique ne serait pas encore en chemin pour devenir une communauté régionale, le quatrième niveau de régionalité, qui se réfère au processus où la région devient un sujet actif avec une identité distincte, une légitimité, un pouvoir de prise de décision, et une société civile régionale. Plusieurs obstacles apparaissent d'emblée pour définir l'Arctique comme une région au sens fort.

Le travail du géographe François Taglioni (2004) sur l'Océanie insulaire permet de mettre en parallèle de nombreux éléments communs avec l'Arctique dans les difficultés que rencontre l'intégration régionale au sens fort définie plus haut. D'abord, la fragmentation des territoires entre océans et terre ferme, dont découle une discontinuité des territoires et pour beaucoup leur enclavement. Les questions climatiques et géographiques ensuite constituent un obstacle, dont découle un manque de communication entre les différentes parties de la région : une région advient aussi lorsque les infrastructures, les systèmes de transports, les formes de mobilités sont progressivement intégrées (Paasi, 1991). Aucun vol long-courrier n'existe par exemple 
entre deux villes arctiques, obligeant à des escales systématiques par les capitales ou plus grandes villes situées dans le sud. À cela s'ajoutent des disparités de développement entre certains pays, que l'on retrouve en Arctique avec les différences économiques, culturelles, sociales, politiques entre des pays aussi différents que la Norvège et la Russie, respectivement $1^{\text {er }}$ et $49^{\text {ème }}$ au classement des IDH mondiaux. Tous ces critères parmi d'autres font que si la première définition de la région dans la typologie de Hettne et Söderbaum peut correspondre à l'Arctique, les conditions pour répondre aux autres définitions ne sont pas complètement remplies.

Difficilement définissable par des pratiques unifiées, nous allons donc voir que l'Arctique apparaît davantage comme un outil politique. La région serait avant tout performative, et il se pourrait que l'Arctique comme région advienne au fur et à mesure qu'il est pensé, délimité, revendiqué, contesté, sujet et objet de politiques régionales.

\section{L'intégration régionale, un outil politique venu « du haut » pour mettre fin à la fragmentation de la Guerre froide}

Il convient à ce titre de nous intéresser aux questions qui ont présidé à la naissance et à la définition de la coopération politique en Arctique, et notamment au Conseil de l'Arctique. Aux origines du processus de coopération régionale se trouve la volonté collective de protéger un environnement fragile et mis à mal par le développement industriel et militaire de la Guerre froide. L'année 1987 marque un tournant dans la géopolitique arctique, avec un célèbre discours de Mikhaïl Gorbatchev qui brosse les premiers éléments du paysage actuel de la coopération. Dans le contexte de la "glasnost» et de la "perestroïka ", l'homme d'État soviétique expose lors d'un discours prononcé dans la ville arctique de Mourmansk ses ambitions pour une politique arctique de coopération et de paix. Il présente six propositions pour l'Arctique, dont celle de « coopérer en matière de protection environnementale ». Ce discours long de 24 pages (Gorbatchev, 1987) se conclut sur plusieurs propositions de normalisation des relations internationales en Arctique, y compris un plan de protection de l'environnement arctique qui est repris par le gouvernement finlandais.

La pollution environnementale de l'Arctique était devenue un sujet d'inquiétude pour les autorités finlandaises à la suite de la prise de conscience de la pollution de régions septentrionales du pays, contaminées par les industries minières soviétiques de la péninsule de Kola (Oude Elfernink, 1992). À l'issue d'un cycle de réunions connu comme "processus de Rovaniemi », les ministres des huit pays circumpolaires adoptent en juin 1991 l'«Arctic Environmental Protection Strategy» (AEPS) qui crée un forum scientifique dédié à la coopération pour la protection de l'environnement arctique. L'AEPS se donne la moins épineuse des propositions de Mikhaill Gorbatchev comme fin, c'est-à-dire protéger l'environnement et l'écosystème arctiques (AEPS, 1991). Dans les faits, l'AEPS a limité ses activités à des activités techniques et scientifiques : la collecte de données, la recherche et l'échange d'informations et la mise en place de recommandations environnementales. L'organisation n'a pas le pouvoir de mettre en œuvre une structure juridique. Les attentes de certains acteurs pour la mise en place de mécanismes solides en réponse aux problèmes environnementaux n'ont jamais été comblées, l'impact en matière de protection de l'environnement demeurant modeste (Tennberg, 2017). 


\section{L'environnement comme prétexte à la construction d'une région politique?}

scientifique au sein des groupes de travil. Ces derniers formulent des recommandations fondées sur l'imbrication du travail de représentants scientifiques et autochtones des huit pays arctiques, d'agences gouvernementales, de chercheurs et d'experts internationaux. L'environnement et la recherche scientifique sont donc la pierre angulaire de la coopération fonctionnelle arctique, en ce qu'ils ont été désignés comme principal point de convergence entre tous les États pour envisager la coopération sans trop engager des questions de souveraineté. Mais bien plus qu'un point d'intérêt commun, ces domaines nous paraissent surtout être un alibi politique, en tant que plus petit dénominateur commun d'entente entre les huit États possédant des territoires au nord du cercle polaire.

\section{La région, un outil avant tout géopolitique?}

Dans un contexte de politisation accrue de la région, la construction de la région arctique devient pour les États arctiques un moyen d'exclure certains acteurs: organisations autochtones et acteurs extérieurs sont progressivement poussés hors des instances décisionnelles de la région. Ces derniers cherchent alors à élargir les limites du périmètre de décision à travers de nouvelles formes de gouvernance. 


\section{L'instrumentalisation stratégique des structures de gouvernance régionale par les États : la région comme outil pour fermer le processus de décision politique}

Une des tendances politiques les plus frappantes en Arctique est sans nul doute l'intérêt mondial récent pour la région. L'ensemble des enjeux commerciaux et économiques attribués à la région attisent les intérêts de pays du monde entier (Lasserre, 2010): possibilité de routes maritimes internationales, zones de pêche élargies, ressources minérales et pétrolières devenues plus accessibles. Dans ce contexte en évolution, nous observons que les États arctiques veulent mieux maittriser le processus de gouvernance régionale.

À l'origine des processus de représentation politique en Arctique, les acteurs étatiques arctiques s'étaient attelés avec beaucoup d'application à effacer, voire refouler le politique en occultant les ressorts idéologiques et symboliques de la représentation. À ce titre, l'institutionnalisation d'une place accordée aux populations autochtones au sein $\mathrm{du}$ Conseil de l'Arctique, l'apaisement de la contestation par son institutionnalisation, peut être vue comme une manœuvre politique de la part des États (Escudé, 2019). Cette analyse rejoint les critiques selon lesquelles le processus de gouvernance régionale se focaliserait sur l'efficacité technique aux dépens de l'efficience démocratique (Kazancigil, 2010).

26 Les intérêts politiques sont ainsi explicitement évacués au sein du Conseil de l'Arctique qui se concentre sur des sujets techniques. Cependant, les enjeux éminemment politiques entre les échelles de représentation ressurgissent. On observe en effet un accroissement des acteurs qui veulent s'impliquer en Arctique, avec un nombre qui va au-delà des États et des institutions dans une forme de " polyphonie " politique (Albert \& Vasilache, 2017, p. 9). Ces acteurs peuvent agir dans la région de multiples manières : scientifiques, économiques, commerciales. Le seul moyen cependant proprement politique est d'acquérir un statut d'observateur au sein du Conseil de l'Arctique.

Même si un compte exact est difficile à connaître, il y aurait environ actuellement seize candidatures pour un statut d'observateur, parmi lesquelles se trouvaient la Grèce, la Turquie, la Mongolie, mais également Greenpeace, l'Association des Producteurs de Pétrole et de Gaz ou encore la National Geographic Society (Rosen, 2016). Le graphique ci-dessous illustre l'intérêt international pour la région arctique. Ce document présente l'entrée des nouveaux observateurs par type (État, Organisation interparlementaire, ONG) au fil du temps. 
Figure 1. La croissance du nombre d'observateurs au Conseil de l'Arctique depuis sa création.

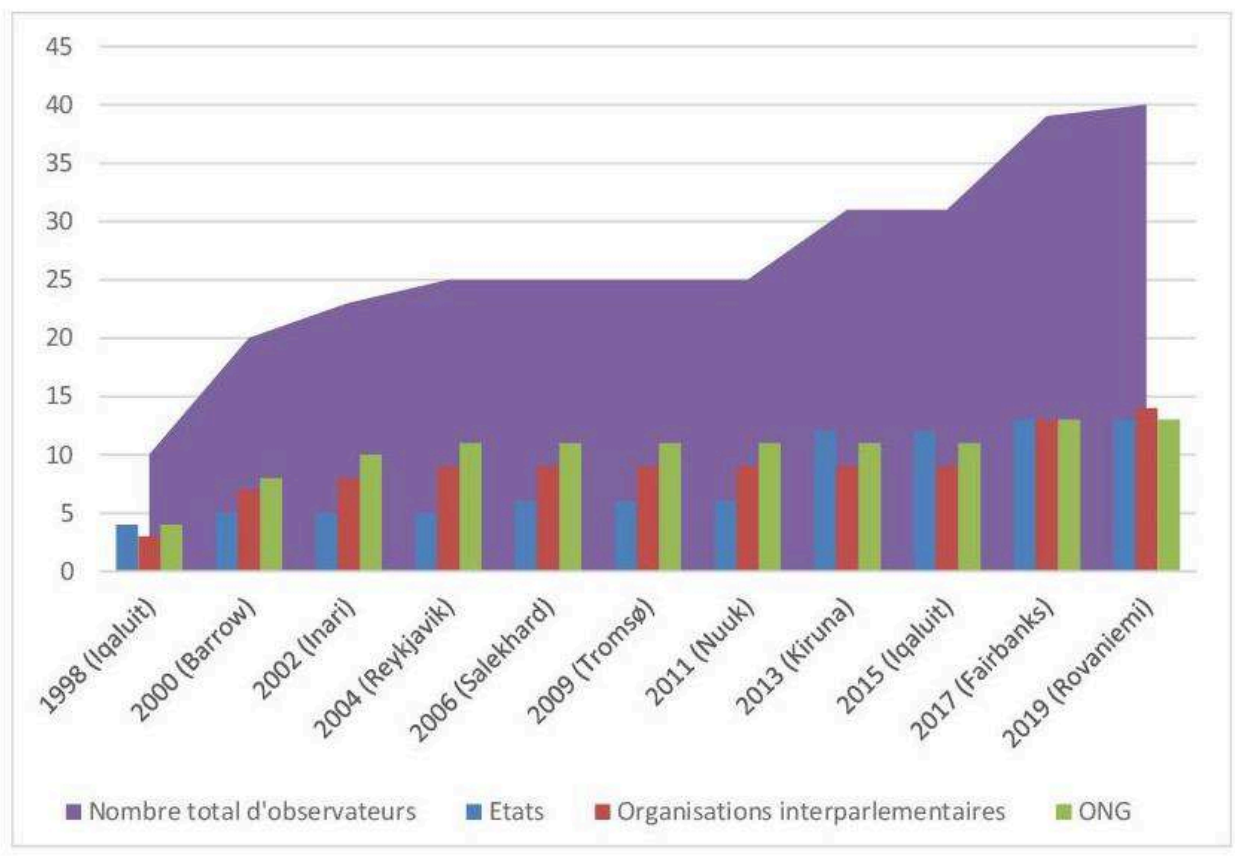

En 2013, le nombre d'observateurs étatiques a doublé, avec l'arrivée notable de nombreux observateurs asiatiques : Corée du Sud, Chine, Japon, Inde et Singapour aux côtés de l'Italie. La population du monde représentée au sein du Conseil de l'Arctique est à présent de $60 \%$, traduisant l'internationalisation de l'intérêt pour la région. Nous pouvons voir avec le tableau ci-dessous le détail de l'entrée des États observateurs dans le temps depuis la création du Conseil.

Tableau 1. Tableau récapitulatif de l'accès au statut d'observateur étatique au Conseil de l'Arctique.

\begin{tabular}{|l|l|l|l|}
\hline Date d'entrée & Pays président & Lieu de la réunion ministérielle & État observateur \\
\hline 1998 & Canada & Iqaluit & $\begin{array}{l}\text { Allemagne } \\
\text { Pays Bas } \\
\text { Pologne } \\
\text { Royaume-Uni }\end{array}$ \\
\hline 2000 & États-Unis & Barrow & France \\
\hline 2002 & Finlande & Inari & \\
\hline 2004 & Islande & Reykjavik & Espagne \\
\hline 2006 & Russie & Salekhard & \\
\hline 2009 & Norvège & Tromsø & \\
\hline 2011 & Danemark & Nuuk & \\
\hline
\end{tabular}




\begin{tabular}{|l|l|l|l|}
\hline 2013 & Suède & Kiruna & $\begin{array}{l}\text { Corée } \\
\text { Chine } \\
\text { Italie } \\
\text { Japon } \\
\text { Inde } \\
\text { Singapour }\end{array}$ \\
\hline 2015 & Canada & Iqaluit & \\
\hline 2017 & États-Unis & Fairbanks & Suisse \\
\hline 2019 & Finlande & Rovaniemi & \\
\hline
\end{tabular}

Malgré l'intérêt croissant pour le statut d'observateur au Conseil de l'Arctique, les États membres montrent de plus en plus d'importantes réticences à l'octroyer. Lors de la réunion ministérielle de Rovaniemi, en mai 2019, un seul observateur a été accepté, il s'agit de l'Organisation Maritime Internationale, organe de l'ONU.

Lors de la réunion ministérielle de 2013 à Kiruna, les règles de procédure pour l'admission des observateurs ont été revues de manière plus stricte (Arctic Council, 2013b), publiées à l'occasion dans un «Arctic Council Observer manual» qui accompagne des règles de procédures réactualisées (Arctic Council, 2013a). Trois éléments notables ont été ajoutés. D'une part, la contribution totale de tous les observateurs à un projet de recherche ne peut pas dépasser celle des États arctiques (article 38). D'autre part, chaque observateur doit renouveler son intérêt tous les quatre ans et peut voir potentiellement sa participation refusée. Enfin, les candidats au statut d'observateur doivent reconnaître la souveraineté des États arctiques dans la région, prouver qu'ils respectent les valeurs des peuples autochtones et qu'ils sont prêts à les soutenir financièrement, manifester leurs «intérêts " pour l'Arctique - un critère délibérément vague qui laisse le champ libre aux États membres pour accepter ou refuser une candidature.

31 La réunion ministérielle de Kiruna correspond également à une rupture puisqu'à partir de 2013, les drapeaux des observateurs ne sont plus autorisés sur la table, ce qu'un diplomate français interrogé analyse comme une «ultra-politisation » et une priorité donnée à la coopération entre les acteurs de la région aux dépens de l'inclusion des acteurs extérieurs (Entretien avec un diplomate français 5, 3/07/2018). Cette politisation se traduit par une exclusion progressive de certains membres observateurs du Conseil de l'Arctique, qui pouvaient ainsi prendre la parole à côté des ministres des États arctiques jusqu'en 2011 et se sont trouvés relégués à un statut purement passif d'observateurs (Entretien avec un diplomate français 5, 3/07/2018).

Cet ensemble de nouvelles règles renforce donc la souveraineté des États du Conseil de l'Arctique. Alors que le Conseil se veut une instance de "développement de bonne gouvernance », cela reste un "club huppé » (Entretien avec un diplomate français 2, 10/01/2018). Un autre diplomate nous a confié la même idée en des termes différents : les observateurs se voient donc relégués «à la table des enfants " (Entretien avec un diplomate français 4, 27/06/2018), tandis que les huit États membres parlent entre «adultes" des choses sérieuses. Michel Rocard, premier Ambassadeur français des 
pôles, qualifiait d'ailleurs le Conseil de l'Arctique de «syndicat de copropriétaires ", où les acteurs extérieurs n'auraient pas de place.

Dans un discours très remarqué à la veille de la $11^{\mathrm{ème}}$ réunion ministérielle à Rovaniemi en mai 2019, le secrétaire d'État états-unien Mike Pompeo pointait l'action menaçante de la Chine en Arctique (Pompeo, 2019) : «Beijing claims to be a "Near-Arctic State", yet the shortest distance between China and the Arctic is 900 miles. There are only Arctic States and Non-Arctic States. No third category exists, and claiming otherwise entitles China to exactly nothing ». Cette déclaration faisait référence à la politique arctique de la Chine lancée en 2018, où elle se définissait elle-même comme "near Arctic state " (The State Council - The people's Republic of China, 2018).

Nous observons donc depuis 2013 la volonté des États circumpolaires de limiter l'implication d'acteurs extérieurs et de circonscrire les limites strictes du périmètre de décision au cercle polaire arctique. Les nouvelles règles du Conseil de l'Arctique peuvent être interprétées comme une volonté des Etats arctiques de renforcer leur exclusivité dans la gouvernance, d'affirmer leur contrôle dans les affaires arctiques et de réaffirmer leur souveraineté. En même temps, la création du statut d'observateur permet de limiter le rôle politique des États non arctiques à un statut passif d'observateur - et non d'acteur politique. L'inclusion des observateurs dans le processus de gouvernance peut être interprétée comme un moyen de verrouiller leur action. Ils sont inclus d'une certaine manière, mais ils sont condamnés à l'impuissance politique au sein de la principale arène politique arctique.

\section{Une région en extension sous la pression géopolitique des nouveaux acteurs}

35 Face à la restriction de leur rôle politique au sein des instances traditionnelles de gouvernance, les acteurs exclus inventent alors de nouvelles formes pour prendre part aux décisions régionales. Cela illustre l'élargissement du cadre géographique de l'Arctique avec une extension auréolaire politique et géographique. L'exemple de la conférence internationale Arctic Circle est représentatif à cet égard. Fondée en 2013 à Reykjavik à l'initiative de l'ancien premier Ministre islandais ólafur Grímsson, cette conférence a donné lieu à des forums parallèles au Québec (2016), en Écosse (2017), et même à Singapour (2015) ou à Shangaï (2019).

36 Les conférences et forums sont des plateformes privilégiées de l'expression d'acteurs ordinairement mis de côté de la coopération formelle en Arctique, dans le temps court de leur durée (3-5 jours). Ces rencontres se font à travers des réunions plus informelles ou bien des sessions en petit comité. Que ce soit pour les entreprises, les États non riverains ou encore les gouvernements infranationaux, ils permettent une coopération plus ouverte. Le forum Arctic Circle permet à des États non-circumpolaires mais également à des acteurs non étatiques, comme le gouvernement provincial du Québec de s'impliquer dans la prise de décision politique arctique: "À Arctic Circle, nous sommes très impliqués, car on peut agir en tant que Québec" (Entretien avec un diplomate québécois 2, 4/06/2018).

37 La structure même du forum Arctic Circle est née de l'implication du gouvernement du Québec au sein d'une initiative islandaise, deux acteurs ayant en commun la volonté de s'impliquer dans une région où leur rôle politique est minoré : le Québec car étant une province canadienne, l'Islande car n'étant pas un État riverain et demeurant le plus 
petit pays arctique avec ses 350000 habitants. Lors de l'Arctic Circle 2014, devant plus de 2000 participants de 50 pays différents, le Premier ministre du Québec Philippe Couillard a affirmé l'engagement du Québec, affirmant «We want, and we will be part of the dialogue on Arctic and Nordic issues " (Delo, 2017). Jouer un rôle dans la formation d'une politique arctique a fait partie des efforts du Québec pour réclamer une voix sur la scène internationale. Les mots de Philippe Couillard affirment la position de la province au niveau infraétatique dans les questions arctiques, et illustre la détermination du Québec à demander un siège à la table des décisions arctiques. De manière révélatrice, le premier Ministre québécois est la seule personnalité canadienne à prendre part au Conseil Honoraire d'Arctic Circle.

Ce format de décision plus informel permet au gouvernement du Québec d'être impliqué dans la gouvernance régionale alors même qu'il est exclu officiellement du Conseil de l'Arctique - et ce malgré le caractère arctique de son territoire du Nunavik. En 2014, 2015, puis 2016, le premier Ministre du Québec s'est rendu aux assemblées. Comme nous l'explique un diplomate québécois, le Québec est l'un des seuls gouvernements à envoyer une personnalité d'aussi haut rang. La raison pour lui est que "les entités étatiques ressentent moins le besoin de s'impliquer, elles ont déjà leur forum qui est le Conseil de l'Arctique " (Entretien avec un diplomate québécois 2, 4/06/2018).

Avec cet exemple pris parmi les acteurs non-étatiques traditionnellement exclus du Conseil de l'Arctique, nous pouvons observer une stratégie de contournement des instances officielles de gouvernance régionale. Alors que la région est un outil politique utilisé par les États circumpolaires pour restreindre le processus de prise de décision, la stratégie québécoise est révélatrice de la réaction des acteurs exclus pour élargir le périmètre de décision au sein de nouvelles formes de gouvernance.

\section{Une région floue révélée par les stratégies des gouvernances multiniveaux}

Nous allons à présent développer la manière dont les stratégies explicites ou implicites des gouvernances multiniveaux révèlent une région arctique à la forme particulière.

\section{De la gouvernance multiniveau à la gouvernance réticulaire de la région arctique qui permet l'intégration régionale}

41 Plus que l'emboîtement des échelles, c'est l'étude de leur interaction et de leur interdépendance qui doit être mise en exergue pour comprendre les dynamiques de l'espace politique contemporain (Rosière, 2009). En Arctique, l'intégration régionale se fait donc à travers une multitude d'initiatives politiques à toutes les échelles de décision et d'application, qui produit une gouvernance multiniveau, définie comme un "système de négociation continue entre les gouvernements nichés à divers niveaux territoriaux » (Marks, 1993, p. 392).

La façon la plus commune d'examiner les niveaux est de les considérer comme une hiérarchie imbriquée d'espaces bornés de différentes tailles et à des niveaux différents (local, régional, national, mondial). Or, les théoriciens des échelles ont souvent critiqué le concept de gouvernance multiniveau car il insiste sur la hiérarchie emboitée aux 
dépens de l'analyse d'un réseau dense et enchevêtré et seulement partiellement hiérarchisé. Une autre façon de conceptualiser les niveaux est donc de les comprendre non comme des couches disjointes d'espaces bornés et ordonnés hiérarchiquement du local au global, mais comme des caractéristiques réticulaires (Mamadouh, van der Wusten, 2009). On peut alors comprendre le niveau arctique comme un réseau politique, incluant des réseaux d'acteurs, non forcément bornés par des domaines territoriaux: le rôle des populations autochtones à cet égard est particulièrement prégnant en tant qu'acteur transnational. En Arctique, les interactions entre les diverses sphères, qui ne sont ni statiques ni imperméables, forment et coconstruisent un système de coopération régionale.

Le tableau 2 rassemble les principales organisations et forums en Arctique et met en exergue leur diversité notamment dans les territoires et les niveaux de décisions impliquées.

Tableau 2. Comparaison structurelle des organisations et forums en l'Arctique.

\begin{tabular}{|c|c|c|c|c|c|c|}
\hline Nom & $\begin{array}{l}\text { Date de } \\
\text { création }\end{array}$ & $\begin{array}{l}\text { Ville } \\
\text { accueillant } \\
\text { le } \\
\text { secrétariat }\end{array}$ & Objectif & $\begin{array}{l}\text { Structures } \\
\text { subordonnées }\end{array}$ & Membres & $\begin{array}{l}\text { Niveau de } \\
\text { décision }\end{array}$ \\
\hline $\begin{array}{l}\text { Conseil } \\
\text { Nordique }\end{array}$ & 1952 & \multirow{2}{*}{$\begin{array}{l}\text { Secrétariat } \\
\text { existant de } \\
\text { depuis } \\
1972, \quad \text { à } \\
\text { Copenhague }\end{array}$} & $\begin{array}{l}\text { Organiser une } \\
\text { coopération } \\
\text { interparlementaire } \\
\text { entre les pays } \\
\text { membres }\end{array}$ & \multirow{2}{*}{$\begin{array}{l}20 \text { groupes de } \\
\text { travail } \\
20 \\
\text { « coopération } \\
\text { bodies » } \\
\text { (centres, } \\
\text { bureaux, } \\
\text { fondations } \\
\text { etc.) }\end{array}$} & \multirow{2}{*}{$\begin{array}{l}\text { Islande, } \\
\text { Danemark, } \\
\text { Norvège, } \\
\text { Suède, Finlande }\end{array}$} & \multirow{2}{*}{ Étatique } \\
\hline $\begin{array}{l}\text { Conseil } \\
\text { Nordique des } \\
\text { ministres }\end{array}$ & 1971 & & $\begin{array}{l}\text { Organiser une } \\
\text { coopération entre } \\
\text { des ministres de } \\
\text { chaque pays sur } \\
\text { un sujet précis }\end{array}$ & & & \\
\hline $\begin{array}{l}\text { West Nordic } \\
\text { Council* }\end{array}$ & 1985 & 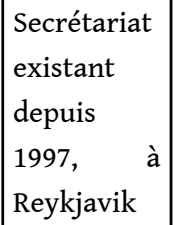 & $\begin{array}{lrr}\text { Promouvoir } & \text { les } \\
\text { intérêts } & \text { et } \\
\text { préserver } & \text { les } \\
\text { ressources } & \text { de } & \text { la } \\
\text { région } & & \end{array}$ & & \begin{tabular}{|lr} 
Parlements & et \\
Gouvernements \\
du & Groenland, \\
Îles & Féroé, \\
Islande &
\end{tabular} & $\begin{array}{l}\text { Étatique, } \\
\text { infra- } \\
\text { étatique }\end{array}$ \\
\hline
\end{tabular}




\begin{tabular}{|c|c|c|c|c|c|c|}
\hline $\begin{array}{l}\text { Forum } \\
\text { Nordique }\end{array}$ & 1991 & & 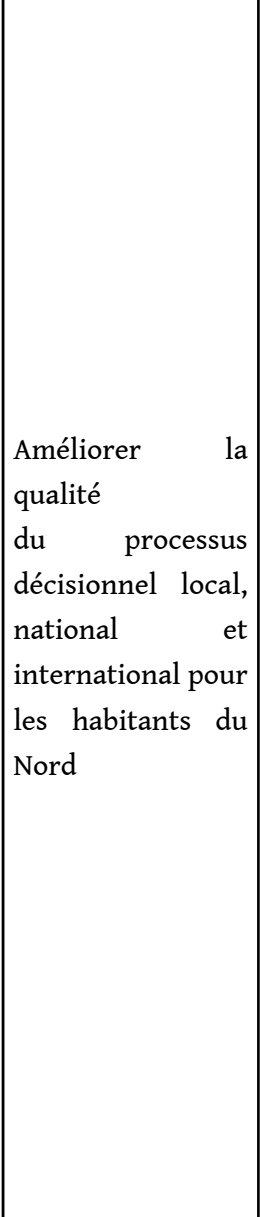 & & $\begin{array}{l}13 \\
\text { gouvernements } \\
\text { infranationaux : } \\
\text { Akureyri } \\
\text { (Islande), } \\
\text { Alaska (États- } \\
\text { Unis), Province } \\
\text { de Gangwon } \\
\text { (Corée du Sud), } \\
\text { Krai de } \\
\text { Kamtchakta, de } \\
\text { Khabarovsk, de } \\
\text { Krasnoyarsk, de } \\
\text { Primorsky, } \\
\text { Districts } \\
\text { autonomes de } \\
\text { Khanty - } \\
\text { Mansiysk, } \\
\text { Chukotka, } \\
\text { Nenetskiy, } \\
\text { Yamal - Nenets, } \\
\text { Oblast de } \\
\text { Magadan, } \\
\text { République de } \\
\text { Sakha (Russie), } \\
\text { Laponie } \\
\text { (Finlande) }\end{array}$ & $\begin{array}{l}\text { Infra- } \\
\text { étatique }\end{array}$ \\
\hline $\begin{array}{l}\text { Conseil des } \\
\text { États de la mer } \\
\text { Baltique }\end{array}$ & 1992 & \begin{tabular}{|l} 
Secrétariat \\
depuis \\
1998, à \\
Stockholm
\end{tabular} & $\begin{array}{l}\text { La protection de } \\
\text { l'environnement, } \\
\text { la coopération } \\
\text { économique et les } \\
\text { questions sociales }\end{array}$ & $\begin{array}{l}\text { Baltic 21, } \\
\text { HELCOM } \\
\text { (Helsinki } \\
\text { Commission } \\
\text { on Baltic } \\
\text { pollution), } \\
\text { Euro-faculty, } \\
\text { «task } \\
\text { forces », } \\
\text { groupes de } \\
\text { travail, } \\
\text { groupes } \\
\text { d'experts }\end{array}$ & $\begin{array}{l}\text { Onze États de la } \\
\text { région baltique, } \\
\text { Commission } \\
\text { européenne }\end{array}$ & $\begin{array}{l}\text { Étatique, } \\
\text { supra- } \\
\text { étatique }\end{array}$ \\
\hline $\begin{array}{l}\text { Comité des } \\
\text { Parlementaires } \\
\text { de la Région } \\
\text { Arctique } \\
\text { (CPRA) }\end{array}$ & 1993 & & $\begin{array}{lr}\text { Réunir les } \\
\text { parlementaires } \\
\text { pour soutenir la } \\
\text { création } \\
\text { Conseil du } \\
\text { l'Arctique, puis } \\
\text { l'accompagner } \\
\text { dans son travail }\end{array}$ & & $\begin{array}{l}\text { Huit États de } \\
\text { l'Arctique, } \\
\text { Parlement } \\
\text { européen, des } \\
\text { représentants } \\
\text { des peuples } \\
\text { autochtones }\end{array}$ & $\begin{array}{l}\text { Étatique, } \\
\text { supra- } \\
\text { étatique }\end{array}$ \\
\hline
\end{tabular}




\begin{tabular}{|c|c|c|c|c|c|c|}
\hline $\begin{array}{l}\text { Conseil euro- } \\
\text { arctique de la } \\
\text { mer de Barents } \\
\text { (BEAR) }\end{array}$ & 1993 & $\begin{array}{l}\text { Secrétariat } \\
\text { depuis } \\
2007, \quad \text { à } \\
\text { Kirkenes }\end{array}$ & $\begin{array}{l}\text { Promouvoir la } \\
\text { coopération } \\
\text { autour du } \\
\text { développement } \\
\text { économique et } \\
\text { social }\end{array}$ & $\begin{array}{l}\text { Regional } \\
\text { Council (of } \\
\text { provinces) } \\
\text { Regional } \\
\text { Committee, } \\
17 \text { groupes de } \\
\text { travail }\end{array}$ & $\begin{array}{l}\text { Cinq pays du } \\
\text { Conseil } \\
\text { nordique, } \\
\text { Russie, } \\
\text { Commission } \\
\text { européenne }\end{array}$ & $\begin{array}{l}\text { Étatique, } \\
\text { supra- } \\
\text { étatique }\end{array}$ \\
\hline $\begin{array}{ll}\text { Conseil de } \\
\text { l'Arctique }\end{array}$ & 1996 & $\begin{array}{l}\text { Secrétariat } \\
\text { depuis } \\
2013, \quad \text { à } \\
\text { Tromsø }\end{array}$ & & $\begin{array}{ll}\text { Six } & \text { groupes } \\
\text { de } & \text { travail, } \\
& \text { task forces } ~\end{array}$ & $\begin{array}{l}\text { Huit États de } \\
\text { l'Arctique, } \\
\text { représentants } \\
\text { autochtones, } \\
\text { Observateurs } \\
\text { (ONG, } \\
\text { Institutions } \\
\text { autres, États) }\end{array}$ & Étatique \\
\hline $\begin{array}{l}\text { Conseil de } \\
\text { coopération } \\
\text { Nord- } \\
\text { Atlantique }\end{array}$ & 1996 & $\begin{array}{l}\text { Secrétariat } \\
\text { à } \\
\text { Tórshavn, } \\
\text { Îles Féroé }\end{array}$ & $\begin{array}{ll}\text { Promouvoir } & \text { le } \\
\text { développement } & \\
\text { durable de la } & \\
\text { région } & \end{array}$ & & \begin{tabular}{|l|} 
Groenland, \\
Islande, Îles \\
Féroé, littoral \\
de la Norvège
\end{tabular} & $\begin{array}{l}\text { Étatique, } \\
\text { Subétatique }\end{array}$ \\
\hline $\begin{array}{l}\text { Dimension } \\
\text { nordique de } \\
\text { l'Union } \\
\text { européenne }\end{array}$ & 1997 & & $\begin{array}{l}\text { Former un cadre } \\
\text { commun pour } \\
\text { favoriser } \\
\text { l'intégration } \\
\text { économique et les } \\
\text { questions de } \\
\text { développement } \\
\text { durable }\end{array}$ & & $\begin{array}{l}\text { Union } \\
\text { européenne, } \\
\text { Russie, } \\
\text { Norvège, } \\
\text { Islande }\end{array}$ & $\begin{array}{l}\text { Étatique, } \\
\text { supra- } \\
\text { étatique }\end{array}$ \\
\hline "Arctic Five" & 2008 & & $\begin{array}{l}\text { Bloquer tout } \\
\text { nouveau régime } \\
\text { juridique } \\
\text { international de } \\
\text { gestion de l'océan } \\
\text { Arctique }\end{array}$ & & $\begin{array}{lr}\text { Cinq } & \text { États } \\
\text { riverains } & \text { de } \\
\text { l'océan } & \\
\text { Arctique } & \end{array}$ & Étatique \\
\hline
\end{tabular}

* Nommé d'abord « West Nordic Council of Parliamentarian Cooperation ».

Nous pouvons voir dans le foisonnement de ces instances officielles de décision que la gouvernance arctique est davantage enchevêtrée que simplement "multiniveaux ». La région est issue d'un ensemble de pratiques, de discours, de relations et de réseaux traversant les frontières et les échelles, et qui peuvent avoir des origines diverses dans le temps et l'espace mais qui sont assemblées et connectées dans des contextes politiques donnés (Paasi, 2010). Dans cette perspective, et avec l'Union Européenne en outil de comparaison, l'Arctique "ne serait en effet pas une échelle de plus qui viendrait s'ajouter aux autres dans un système politique hiérarchiquement bien ordonné, mais un nouveau type de configuration scalaire de gouvernance où chaque échelle est en relation plurielle avec les autres" (Yann Richard et Cristina D’Alessandro, 2018). Pour suivre la réflexion de Virginie Mamadouh et Herman Van der 
Wusten sur l'échelle européenne (2008), le niveau arctique n'est pas seulement une nouvelle échelle de gouvernement, il inclut de nouvelles relations entre les différents niveaux. Cela rappelle également le concept de formations socio-spatiales inventé par Guy Di Méo, « unités territoriales de taille intermédiaire [...] constituant des ensembles emboîtés et hiérarchisés établissant entre eux des liens d'interdépendance et formant des organismes de plus en plus complexes » (Di Méo, 1985). La région arctique ne serait pas une région par simple agrégation verticale de territoires d'un niveau hiérarchique inférieur, mais une région par intersection horizontale, faite de la superposition des niveaux comme le montre le schéma ci-dessous, le cœur géométrique et fonctionnel de la région étant l'océan Arctique.

Figure 2. Essai de typologie de construction politique régionale.
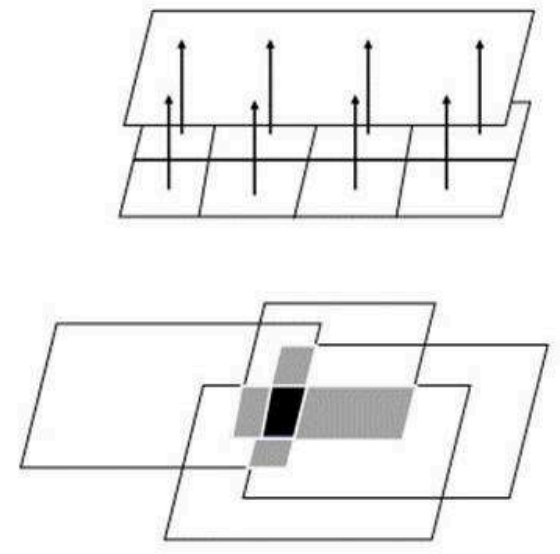

Cas 1 : Région par agrégation Interactions scalaires constitutives (verticales)

Cas 2 : Région par intersection Interactions scalaires collaboratives (horizontales) ne pouvons pas définir la région arctique unir - la complexité des définitions de l'Arctique l'illustre bien. Pour définir l'Arctique comme région à travers la seule appartenance à la région politique, il faudrait agréger les différents pays membres du Conseil de l'Arctique et obtenir ainsi un ensemble aux limites nettes. Mais les limites de l'ensemble régional pourront potentiellement ne pas prendre la forme classique des limites linéaires.

La région arctique ne peut donc pas être définie comme une région par simple agrégation verticale de territoires d'un niveau hiérarchique inférieur (cas 1) mais elle est bien plutôt une région qui apparaît par intersections horizontales (cas 2). Le noir correspondrait alors à une appartenance totale, le gris à une indétermination, le blanc à une absence d'appartenance. Comme cette figure le montre, entre "arctique » et «non arctique» existe une frange pour laquelle la qualification de l'appartenance devient incertaine. Ces dégradés s'observent dans les territoires politiques arctiques, étatiques ou non, qui sont impliqués dans plusieurs initiatives régionales de coopération. Si cette fonction d'appartenance est égale à " 100 », l'unité élémentaire spatiale considérée est toujours incluse dans une région arctique, sans pour autant que cette région soit toujours la même. Dans la plupart des cas, la fonction d'appartenance de l'unité élémentaire est comprise dans l'intervalle $[0 ; 100]$ qui traduit une forme d'intensité d'intégration à l'Arctique. Seul le cœur (l'espace océanique central) est un sous-espace homogène, les franges devront être considérées comme des sortes de mosaïques traduisant l'inégale appartenance des composantes à l'espace et l'hétérogénéité de ce dernier. 
47 Nous avons montré la position intermédiaire de l'espace arctique sur un plan horizontal (espace de convergence entre des réseaux institutionnels enchevêtrés) et vertical (un niveau situé entre le territoire étatique et l'espace mondial). En ce sens, l'espace arctique est bien construit par des réseaux qui mettent en contact les différents niveaux de la gouvernance et est traversé par des réseaux qui le débordent. En jouant du multi-échelle et du multiniveau, l'espace arctique peut être qualifié de région à partir du moment où il est le produit d'une convergence de réseaux d'acteurs institutionnels.

\section{Le caractère dynamique de l'intégration forme une région floue}

48 Le premier, soulignant que le Nord est à la fois imaginaire et physique, le géographe québécois Louis-Edmond Hamelin expliquait que l'identité même de l'Arctique paraît être réduite à son établissement au-delà de la ligne imaginaire du $66^{\circ}$ parallèle alors que d'autres limites paraissent plus appropriées (Hamelin, 1975). Dans les années 1960, il propose la notion de nordicité pour qualifier ce qui rend l'espace nordique. Refusant toute définition objective, Hamelin définit la nordicité d'un lieu à partir d'éléments biophysiques et socio-économiques. Comprendre le Nord à partir de facteurs humains a constitué un élément tout à fait nouveau pour une région considérée jusqu'alors comme uniquement physique. La nordicité s'exprime en "valeurs polaires » (VAPO) à partir de dix critères. Une fois combinés, ces critères se traduisent en VAPO qui s'additionnent pour déterminer le degré de nordicité d'un espace, le pôle nord ayant la valeur maximale de 1000 VAPO. À partir de lieux référencés, Hamelin trace des isolignes de nordicité en décrivant de nouvelles limites non réductibles à leurs seules latitudes. Les résultats permettent d'identifier différentes régions aux valeurs polaires similaires et ainsi de proposer d'autres lectures du Nord - et par conséquent de l'Arctique. De manière très intéressante, la nordicité est un outil qui permet de démontrer le caractère changeant des Nords puisque certains territoires vivent une "dénordification », du fait de l'augmentation de leur accessibilité, l'apparition de nouvelles villes, ou du fait du changement climatique. Au contraire, l'identification de zones de "pré-Nord» permet de sortir du raisonnement d'une frontière fixe de l'Arctique. Garder à l'esprit la possibilité de limites mouvantes et non définitives de la région nous est utile pour penser la région comme un objet non fini.

Les travaux récents de géographes comme Clarisse Didelon-Loiseau sur la logique floue appliquée à l'étude de la régionalisation du monde sont à cet égard très éclairants (Didelon - Loiseau, 2018). Le rapprochement avec le cas de l'Union européenne se révèle une fois de plus fructueux. Alors que l'Union européenne a une définition claire de ses membres, nombreux sont les chercheurs qui soulignent le caractère flou de ses limites, selon différents indicateurs. Pour les politistes Thomas Christiansen, Fabio Pztito et Ben Tonra, l'Union européenne est entourée de régions qui sont des espaces intermédiaires entre l'intérieur et l'extérieur de la région, qui sont devenus des cibles d'exportation de sa politique, brouillant les limites (2000). Les frontières de l'Europe peuvent être vues comme floues car elles produisent des interfaces ou des espaces intermédiaires entre l'intérieur et l'extérieur de la politique. Plus que «borders », les chercheurs préfèrent le terme de "frontier » qui en anglais a une connotation plus diffuse. Il s'agit de zones mouvantes, perméables et fluides qui permettent le passage. L'idée de "fuzzy borders" souligne l'idée selon laquelle l'UE est entourée par des espaces intermédiaires placés entre le dedans et le dehors, des espaces où elle exporte 
certaines idées politiques et dans lesquels elle a un intérêt croissant. Tous les auteurs conçoivent la région comme un système spatial dont les limites sont mouvantes et floues et à l'intérieur duquel les interdépendances, éventuellement dissymétriques, reposent sur des complémentarités.

C'est bien le cas en Arctique, d'autant plus que cela nous amène à définir une région dont les limites peuvent couper en deux le territoire d'un ou plusieurs États, laissant certaines de leurs parties en dehors du processus. Comme l'écrit Yann Richard: «Cela permet de souligner le caractère dynamique de l'intégration, qui donne forme à des régions dont l'organisation interne et les limites sont en perpétuel mouvement, ainsi que l'existence d'autres dynamiques plus subtiles comme la diffusion de normes et d'institutions par les organisations régionales. Les régions ainsi définies juxtaposent des noyaux durs et des périphéries plus ou moins fragiles et plus ou moins larges, ce qui signifie que leurs limites sont à la fois mouvantes et non linéaires. Ce seraient plutôt des dégradés qui juxtaposent des territoires ou les caractères du noyau dur régional s'effacent progressivement » (Richard, 2018).

51 Ces dégradés s'observent dans les territoires politiques arctiques, étatiques ou non, qui sont impliqués dans plusieurs initiatives régionales de coopération. On pourrait alors définir un gradient "d'arcticité » plus élevé à mesure que la maille territoriale appartiendrait à un nombre plus grand d'organisations politiques arctiques, pour reprendre le tableau 2. Par exemple, la Laponie, l'une des dix-neuf régions finlandaises, est impliquée dans plusieurs organisations régionales : le Conseil nordique, le Conseil de l'Arctique, le Conseil des États de la mer Baltique, le Conseil euro-arctique de la mer de Barents grâce à l'État finlandais, et en plus dans le Forum Nordique en tant que région. Par rapport à l'Alaska, présent uniquement au Conseil de l'Arctique, la Laponie présenterait donc un gradient d'« arcticité », ou encore d'intégration régionale arctique plus fort. En suivant cette logique, l'Alaska présenterait le même niveau d'intégration politique arctique que la province sud-coréenne de Gangwon (Corée du Sud), présente au Forum Nordique. Ces délimitations politiques qui amènent à nous interroger sur le caractère définitif de la région dessinent ainsi une région arctique en « donut », avec un vide correspondant à l'océan et des limites « molles » et floues à ce cercle dans sa partie méridionale.

\section{Conclusion}

52 Cet article a cherché à mettre en évidence la construction d'un nouvel espace régional : l'Arctique. En s'appuyant sur une approche constructiviste, nous avons mis en évidence les interactions entre le système de gouvernance et la consolidation de la région comme espace politique. Ce sont les dynamiques politiques et sociales qui ont permis une définition de la région arctique comme espace politique tout à la fois autonome et intriqué dans un système dynamique global.

Des acteurs des échelles d'actions différentes participent à la construction de la région en convergeant pour renforcer la dimension régionale. L'Arctique apparait comme une unité sociale qui manque de permanence et est tiraillée entre de nombreuses différentes délimitations géographiques. Les territoires se réclamant de l'Arctique ou bien demandant un droit dans la gouvernance politique arctique varient dans l'espace et leur nombre a augmenté. L'existence simultanée de limites mouvantes et de différentes configurations d'acteurs ajoute à ce flou. Le niveau arctique consiste en de 
nombreux acteurs dans de nombreuses configurations, qui convergent également dans une série d'arènes de coopération. La tension étudiée entre deux dynamiques contradictoires, centrifuge et centripète, permet de comprendre que la région est avant tout un processus subjectif, mouvant et rétif aux définitions définitives.

Si l'intégration régionale est un phénomène dynamique dans l'espace et dans le temps et multiforme, la région arctique peut se rétracter et le processus d'intégration prendre fin (Mareï, Richard, 2018). Si Hamelin mettait en évidence un processus de dénordification on pourrait imaginer un processus de dés-arctification de la même manière. À ce titre, le refus historique états-unien de signer une déclaration commune à l'issue de la réunion ministérielle du Conseil de l'Arctique en 2019 pourrait signifier un coup d'arrêt à la volonté commune de construire une région politique.

\section{BIBLIOGRAPHIE}

ACIA (2004), Impact of a warming Arctic: Arctic climate change impact assessment, Cambridge University Press, http://www.acia.uaf.edu/pages/overview.html [consulté le 13 septembre 2017].

AEPS (1991), Arctic Environmental Protection Strategy [s.l.], Rovaniemi, Finland.

ALBERT M., VASILACHE A. (2017), « Governmentality of the Arctic as an international region », Cooperation and Conflict, pp. 1-20.

ALLEN J, MASSEY D. \& COCHRANE A (1998), Rethinking the region, New York, Routledge, 159 p.

ARCTIC COUNCIL (1996), « Ottawa Declaration », https://oaarchive.arctic-council.org/handle/ 11374/85 [consulté le 12 mai 2018].

ARCTIC COUNCIL (2013a) « Arctic Council Observer Manual for Subsidiary Bodies », https:// oaarchive.arctic-council.org/handle/11374/939 [consulté le 13 novembre 2018].

ARCTIC COUNCIL (2013b), « Arctic Council Rules of Procedure », https://oaarchive.arcticcouncil.org/handle/11374/940 [consulté le 29 juin 2018].

BLOOM E. (1999), «Establishment of the Arctic Council », American Journal of International Law, 93, 3. pp. 712-722.

CHRISTIANSEN T., PZTITO F. \& TONRA B. (2000), « Fuzzy Politics Around Fuzzy Borders: The European Union's `Near Abroad' », Cooperation and Conflict, 35, 4, pp. 389-415.

DELO A. (2017), « Does Québec Need the Arctic Council? », World Policy, https://worldpolicy.org/ 2017/09/06/does-quebec-need-the-arctic-council-2/ [consulté le 23 janvier 2019].

DI MÉO G. (1985), « Les formations socio-spatiales ou la dimension infra-régionale en géographie », Annales de géographie, 94, 526, pp. 661-689.

DIDELON-LOISEAU C. (2018), « Découpages du monde : la vaine quête d'un découpage universel », Dictionnaire de la régionalisation du monde, Neuilly-sur-Seine, Atlande, pp. 120-122. 
EINARSSON N., YOUNG O., NYMAND LARSEN J. et al. (eds.) (2004), Arctic Human Development Report, Akureyri, Iceland, Stefansson Arctic Institute, http://link.springer.com/referenceworkentry/ 10.1007/978-94-007-0753-5_104 [consulté le 14 septembre 2017].

ESCUDÉ C. (2019), « Le choc des échelles : la représentation politique des territoires locaux de l'Arctique ", in BRUNET S., LEBON L. \& RICHARD Y. (eds.), Prolifération des territoires et représentations de l'Union européenne, Dossiers des Annales de Droit, Rouen, PURH, pp. 79-97.

GORBACHEV M. (1987), The speech in Murmansk at the ceremonial meeting on the occasion of the presentation of the order of Lenin and the gold star to the city of Murmansk, Murmansk, Russia, 1 October 1987, https://www.barentsinfo.fi/docs/Gorbachev_speech.pdf [consulté le 9 janvier 2020].

HAMELIN L. (1975), Nordicité canadienne, Montréal, Cahiers du Québec (Collection Géographie), Hurtubise HMH, 458 p.

HETTNE B. (2003), « The New Regionalism Revisited », in SÖDERBAUM F., SHAW T.M. (eds.), Theories of New Regionalism, London, Palgrave Macmillan UK, pp. 22-42.

HETTNE B. (1994), « The regional factor in the formation of new world order », in SAKAMOTO Y. (ed.), Global transformation: challenges to the state system, Tokyo, New York, United Nations University Press.

HETTNE B., SÖDERBAUM F. (2000), « Theorising the Rise of Regionness », New Political Economy, 5, 3. pp. 457-472.

KAZANCIGIL A. (2010), La gouvernance pour ou contre le politique ?, Paris, A. Colin, 244 p.

LASSERRE F. (2010), « Géopolitiques arctiques : pétrole et routes maritimes au cœur des rivalités régionales ?», Critique internationale, 49, pp. 131-156.

MAMADOUH V., VAN DER WUSTEN H. (2009), « Echelles et territoires dans le système de gouvernance européen », in ROSIÈRE S., COX K., VACCHIANI-MARCUZZO C. et al. (eds.), Penser l'Espace Politique, Paris, Ellipses Marketing, pp. 288-304.

MAREÏ N., RICHARD Y. (2018), « Pourquoi parler de l'intégration régionale aujourd'hui ? » Dictionnaire de la régionalisation du monde, Neuilly-sur-Seine, Atlande, pp. 21-34.

MARKS G. (1993), « Structural Policy and Multilevel Governance in the EC », in CAFRUNY A.W., ROSENTHAL G.G. \& EUROPEAN COMMUNITY STUDIES ASSOCIATION (eds.), The Maastricht debates and beyond, Boulder, Col., Burnt Mill-Harlow, England, L. Rienner Publishers, Longman, pp. 391-410.

OUDE ELFERINK A. (1992), « Environmental protection in the Arctic - The Rovaniemi process », Marine Pollution Bulletin, 24, 3, pp. 128-130.

PAASI A. (1991), « Deconstructing Regions: Notes on the Scales of Spatial Life », Environment and Planning A., 23, 2, pp. 239-256.

PAASI A. (2010), « Regions are social constructs, but who or what 'constructs' them? Agency in question », Environment and Planning A, 42, 10, pp. 2296-2301.

POMPEO M. (2019), Looking North: Sharpening America's Arctic Focus, Rovaniemi, Finland, https:// www.state.gov/looking-north-sharpening-americas-arctic-focus/ [consulté le 10 juin 2019].

RICHARD Y. (éd.) (2014), La régionalisation du monde : construction territoriale et articulation globallocal, Hommes et sociétés, Paris, Karthala, Tunis, IRMC, 272 p. 
RICHARD Y. (2018), « Nouveau régionalisme : un renouvellement de la recherche sur l'intégration régionale », Dictionnaire de la régionalisation du monde, Neuilly-sur-Seine, Atlande, pp. 184-186.

RICHARD Y., D’ALESSANDRO c. (2018), « Gouvernance : mécanisme de gouvernement de la région et outil d'intégration régionale », Dictionnaire de la régionalisation du monde, Neuilly-sur-Seine, Atlande, pp. 164-167.

ROSEN Y. (2016), « How many observers can Arctic Council handle? », The Independent Barents Observer, https://thebarentsobserver.com/en/arctic/2016/03/how-many-observers-can-arcticcouncil-handle [consulté le 12 décembre 2018].

ROSIÈRE S. (2009), « L'espace politique : quels concepts et quelles échelles ? ", Penser l'espace politique, Paris, Ellipses.

TAGLIONI F. (2014), « La coopération régionale dans l'Océanie insulaire : des processus polymorphes ", Les Cahiers d'Outre-Mer. Revue de géographie de Bordeaux, 57, 225, pp. 53-74.

TENNBERG M. (2017), Arctic Environmental Cooperation : A Study in Governmentality, Taylor \& Francis.

THE STATE COUNCIL - THE PEOPLE'S REPUBLIC OF CHINA (2018), China's Arctic Policy, http:// english.gov.cn/archive/white_paper/2018/01/26/content_281476026660336.htm [consulté le 1 juin 2018].

\section{NOTES}

1. Canada, Danemark, États-Unis, Finlande, Islande, Norvège, Suède, Russie.

\section{RÉSUMÉS}

Dans cet article, nous montrons que la coopération politique instituée «par le haut » en Arctique à la fin de la Guerre froide a mené à une intégration régionale institutionnelle. Cette dernière s'est centrée autour de la protection de l'environnement, qui paraît cependant être avant tout un prétexte politique. Nous comprenons en effet la région comme un outil politique, utilisé par les États circumpolaires pour restreindre de plus en plus le périmètre de décision régional. En retour, ce modèle de gouvernance fermée est remis en cause par de nouveaux acteurs, en particulier fédéraux (Québec) mais aussi étatiques internationaux ou autochtones, qui tentent d'élargir les limites de la région avec de nouvelles formes de gouvernance plus ouvertes. Les stratégies des acteurs qui dessinent une gouvernance multiniveau et enchevêtrée forment une région par intersection aux limites floues et contestées.

In this paper, we aim that it is the political cooperation driven "from above" in the post-Cold War era that has led to visible regional institutional integration in the Arctic. This institutional integration stems from the Arctic state's desire to protect the environment as a pretext for political cooperation. In a context of increased politicization of the region due to the consequences of climate change, the construction of the Arctic region becomes indeed a way for 
the Arctic states to gradually pushed out indigenous organizations and external actors from the decision-making bodies. In return, this model of closed governance is challenged by those who are trying to broaden the boundaries of the region with new and more open forms of governance. Political actors at different scales thus converge to build regional governance that is not only multilevel, but also entangled and creates a region with fuzzy borders.

INDEX

Keywords : region, regional integration, Arctic, Arctic Council, international relations, constructivism, geopolitics, governance, regional cooperation

Mots-clés : région, intégration régionale, Arctique, Conseil de l'Arctique, relations internationales, constructivisme, géopolitique, gouvernance, coopération régionale

\section{AUTEUR}

\section{CAMILLE ESCUDÉ-JOFFRES}

Géographe, chercheuse au Centre de recherches internationales (CERI) de Sciences Po et analyste au Groupe d'Etudes de Géopolitique de l'ENS, camille.escude@sciencespo.fr 